\title{
Factors associated with non-adherence to Antiretroviral Therapy among HIV infected adolescents at Guji zone Health Facilities, South Ethiopia
}

Lalisa Ayele Woldasemayat ( $\sim$ lalisaayele@yahoo.com )

Jimma University

Beshir Ibrahim Jiru

Jimma University

Zerihun Kura Edossa

Jimma University

Research

Keywords: Non-adherence, Antiretroviral Therapy, adolescents, Guji zone, Ethiopia

Posted Date: November 17th, 2020

DOI: https://doi.org/10.21203/rs.3.rs-108419/v1

License: (c) (i) This work is licensed under a Creative Commons Attribution 4.0 International License. Read Full License 


\section{Abstract}

Background: Suboptimal adherence results in inadequate drug exposure and increases the likelihood of viral replication and resistance, limit future therapeutic options. A high level of adherence is very crucial to maximize the usefulness of antiretroviral therapy. This study aimed to examine factors associated with non-adherence to antiretroviral therapy among adolescents in Guji Zone, South Ethiopia.

Methods: Institution based case-control study design was employed on adolescent clients on antiretroviral therapy in the ART clinic of Guji zone health facilities from 26 February to 20 April 2018. Cases and controls were recruited by a consecutive sampling method. Bivariate and multivariable logistic regression was done using SPSS version 20 at $95 \%$ Confidence Interval $(\mathrm{Cl})$ and a precision of $5 \%$.

Results: A total of 297(101 cases and 196 controls) adolescents on ART participated in this study. Being rural residents AOR 1.9(1.1-3.6), Living with unmarried/widowed and separated caregivers AOR 2.1(1.2-3.8), living with unemployed caregivers AOR 4.2(2.0-8.9), not disclosing HIV Status AOR 5(2.8-9.4), having symptom of depression for adolescents AOR 2.7(1.5-4.9), not using reminding methods AOR 2.4(1.2-4.8) and Uses of other drugs besides ART AOR 2.6(1.5-5.0) were found to be independent factors associated with non-adherence to ART among adolescents.

Conclusion:-Residence area, caregiver marital status, sources of income for the family, disclosure status, depression status, uses of reminding methods, and uses of other drugs besides ART were factors associated with non-adherence to antiretroviral therapy among adolescents. Support from peers and family can promote adolescent's adherence, as it buffers the stress associated with the illness, encourages optimism, reduces depression, and improves healthful behaviors. The provision of health education on the importance of treatments and needs for adherence should be encouraged. Uses of reminding methods, continuous monitoring of clients on ART regarding the uses of medications other than antiretroviral therapy is important.

\section{Background}

WHO defines adherence as the extent to which a patient's behavior coincides with medical or patient follows medical instructions (1). Poor adherence to treatment in HIV is extremely complex both in its causes and its capacity to negatively affect patient outcomes, treatment options, and healthcare costs. Achieving such high rates of adherence is very challenging to such patients, because their regimens include multiple, often expensive medications that have complex dosing schedules and may cause food interactions and side effects that result in poor tolerability (2).

Globally, an estimated 2.1 million adolescents are living with HIV in 2016, among which 150 adolescents dying from AIDS-related causes every day. This report emphasizes that between 2000 and 2015, annual AIDS-related deaths declined for all age groups except adolescents, where mortality more than doubled from 18,000 to 41,000 per annual (3).

AIDS is the leading cause of death among adolescents in Africa and the second most common cause of death among adolescents globally (4) Adolescents (10-19 years) continued to be vulnerable, both socially and economically, to HIV infection despite efforts to date. This is particularly true for adolescents especially girls who 
live in settings with a generalized HIV epidemic or who are members of key populations at higher risk for HIV acquisition or transmission through sexual transmission (5).

In sub-Saharan Africa (SSA), adolescent girls and young women accounted for $25 \%$ of new HIV infections among adults. The reason for the vulnerability in sub Saharan region were Harmful gender norms and inequalities, insufficient access to education and sexual and reproductive health services, poverty, food insecurity and violence, are at the root of the increased HIV risk of adolescent girls(6). Two out of three newly infected adolescents aged 15-19 years were girls in SSA. As a reason of successful scale up and effectiveness of antiretroviral therapy (ART), children living with HIV are surviving and growing into adolescence. This increasing population requires ongoing support to remain in care and adhere to ART, as well as to manage the changes related to adolescence (7).

Ethiopian adolescent communities today face many health-related challenges, and their lives vary considerably from those of their parents, grandparents and other relatives. A number of behaviors lead to increased risk of getting HIV/AIDS among adolescents in Ethiopia, were unprotected sexual intercourse and multiple sexual partners (8). Regarding factors related with occurrence of HIV on adolescents, different factors that can lead to the occurrence of HIV/AIDS in adolescents are biological, transactional sex, violence, limited access to education and lack of access to service are some of the factors contributing for acquisition of HIV among adolescents globally (9). Adolescents represented the fastest growing age group of people living with HIV (PLHIV), accounting for $5.9 \%$ of the burden of HIV that underscores the need for greater understanding and suitable adaptation of HIV care and treatment services. Sustaining optimal ART adherence in adolescents (ALHIV) has emerged as a major healthcare challenge, fundamentally due to regimen complexity and adherence efficacy (10).

In Ethiopia studies done on ART Adherence reported different level of adherence ranging from 0-100\%) (11-15) in study done in Addis Ababa, revealed that accounting for average nonadherence rate of $26.5 \%(11)$.Another study in the same area among adolescent reported a self-reported nonadherence level as 20.9\% (12). Although the potential negative effects of poor adherence are known, in patients with HIV, adherence takes on particular importance as adherence may impact not only viral suppression, but also the emergence of permanent treatment resistance. Treatment-related factors, patient-related factors, provider-related factors, and healthcare systemrelated factors may all impact adherence (13). Therefore, the aim of this study was to assess factors associated with non-adherence to ART among adolescents patients in Guji zone Oromia Region,Ethiopia.

\section{Methods}

A case-conrol study was conducted from February 26 up to April 20/2018 at Guji zone Public health facilities which is found at south eastern part of Ethiopia $600 \mathrm{~km}$ away from Addis Ababa. The total estimated population of the Guji zone is approximately 1,432,527, of whom 358,131 are adolescents aged (10-19). In Guji zone there are 4566 peoples receiving ART among these 463 are adolescents aged 10-19 years of age accounting $10.14 \%$ of the total peoples on ART services at ART centers in the zone during study period. Both first line and second line treatment were available in the zone ART centers. Total number of peoples newly infected and enrolled to ART in 2016/7 (2009 E.C) were 753 from these $6.4 \%$ were adolescents of which $89.6 \%$ were girls (14).

\section{Study sample}


Those Adolescent clients on ART for at least three months at the selected ART Care Centers were included in the study.. The sample size of the study was calculated by Epi-Info ${ }^{\text {TM }} 7$ software Statcalc program. Variables that are used to calculate sample size are disclosure status, felt depressed, gender, types of health facility, WHO staging ( 3 and 4), experienced ART side effect, distance of health facility. Sample size was calculated by using double population proportion formula. The expected prevalence of exposure among controls felt depressed $3.3 \%$ and prevalence of cases with exposure felt depressed $13.1 \%$ from study conducted in Malawi considering $5 \%$ level of significance, $80 \%$ power of the study and 2:1 controls to case ratio. Accordingly, the the largest sample size selected for this study involved case 101 and control 196, total 297 adolecents taking ART from documentation durind the study period. Purposively Sampled 6 government health facilities ( 2 hospitals and 4 health centers) based on number of adolescent on ART at health facilities (those having $>5$ clients) were included in this study. From purposively selected health facilities, the total adolescents on ART were four hundred twenty one. Then the calculated sample size were distributed to the selected health facilities using proportional to population size based on total adolescent on ART at each selected clinic. In this allocation, adolescents visited to ART clinic at respective health facilities during data collection period were approached. Based on these the recruited health facilities were Shakiso Health Center with 275 clients(case=67, control=128), Megado Health Center with 54 clients(case=7, control=14), Adola General Hospital with 35 clients(case=8, control=17), Negelle General Hospital with 30 clients (case=13, control=24), Adola Health Center with 21 clients (case $=5$, control=10) and Haraqalo Health Center with 6 clients (case $=1$, control=3) were included based number of adolescents on ART in these facilities during data collection period. Cases: Adolescents taking ART doses those who missed doses completely or single missed dose of 3 or 7 days recall or those who have less than $95 \%$ adherence ( missing $>2$ doses of 30 doses) $(15,16)$

Control: Adolescents taking ART doses those who take doses ( $95 \%$ or more adherence $=$ missing $\leq 2$ doses of 30 days doses or no missed dose on 3 or 7 days recall $(15,16)$

\section{Data collection Procedures}

The data were collected using Semi- structured questionnaire constituted eight items relating to Sociodemographic related factors, including Sex ,Age , Residence, Religion, Marital status ,Educational status, Occupational status, Income. The second section constituted seven questions relating to Psychosocial related factors, including Presence of depression, family member with HIV, Disclosure of HIV status, Stigma and Discriminations, Family support, Alcohol/substance use and perception towards ART. The third section constituted eight questions relating to Disease-related factors, including too many pills, Side effect of the drugs, Scheduling problem, Frequency of daily doses, Length of the treatment, CD4 count, WHO stage, Other comorbidity. The four-section constituted six questions relating to Health care delivery systems related factors, including Healthcare facilities (types), Patient provider's relationship, Health care providers' experience, Health education/information, Provision of privacy, distance of health facility. The adherence questionnaire was prepared from AIDS Clinical Trial Group (ACTG) and by reviewing different literature conducted in Ethiopia and sub-Saharan Africa. The patient health questionnaire (PHQ9) with 9-items on which score ranges from 0 to 27 was used to assess the depression of the study participants. Individuals up on their response categorized into five categories as (no depression, mild depression, moderate depression, moderately severe depression and severe depression) and then dummy coded as not having symptom of depression and having symptom of depression(17). Data were collected by four BSc Nurses and supervised two BSc nurses after two days trained on the objectives, data collection techniques, data quality, and interview techniques for this study. Data collections 
were conducted on the arrival of the client to service provision point in separate ART room for privacy issue and controls were recruited after case. The questionnaire was translated into local languages (Afan Oromo and Amharic) then retranslated back to English to maintain consistency by language experts of both languages. The collected data were checked and cleaned for its completeness before entering into Epidata version 3.1.1 software by principal investigator. Missing values and outlier were checked before analysis by running descriptive analysis.

\section{Data analysis procedures}

After collecting the data were cleaned, coded, and entered to Epidata version 3.1. Data were checked for completeness and uniformity before entry in to Epidata. Analysis was by using SPSS version 20 statistical package. Descriptive analysis was conducted and the result was displayed in the form of tables, charts. Then binary logistic analysis was conducted to identify candidate variables for multivariable analysis. Independent variables having $p$. value $\leq 0.25$ were selected as candidate variables for multivariable logistic regression. Multicollinearity between independent variables was checked by logistic regression standard error coefficient (SE) and checking for variance inflation factors and tolerance. Backward stepwise multivariable logistic regression analysis was conducted in order to identify factors independently associated with the non-adherence. The Hosmer and Lemeshow goodness of fitness of the model were also checked. P-value $\leq 0.05$ was used to decide whether there was statistical significance or not.

\section{Ethical consideration}

The study protocol was reviewed and approved by the Jimma University Research Institutional Review Board(IRB). An official letter of cooperation was obtained from the institute of Health to Zonal Health Offices(Guji Zone) and permission was secured from the Guji Zonal Health Offices directors. Informed verbal consent was obtained and confidentiality was kept by avoiding the patient identifier like name and patient medical record number from questionnaire.

\section{Results}

A total of adolescents who are on ART (101 cases and 196 controls) were participated in the study making the response rate $100 \%$. The mean age of control participants was 14.9 years with SD of 2.8 years while the mean age for case participants was 15 years with SD of 2.8 years. About $58(57.4 \%)$ cases and $119(60.7 \%)$ controls were Females. (table 1).

Table 1: - Socio-demographic Characteristics of Adolescent on ART in Guji zone health facilities, Ethiopia, 2018. 


\begin{tabular}{|c|c|c|c|}
\hline Variables & Categories & $\begin{array}{l}\text { Cases } \\
(\%)\end{array}$ & $\begin{array}{l}\text { Controls } \\
\text { (\%) }\end{array}$ \\
\hline \multirow[t]{2}{*}{ Sex } & Male & $43(42.6 \%)$ & $77(39.3 \%)$ \\
\hline & Female & $58(57.4 \%)$ & $119(60.7 \%)$ \\
\hline \multirow{2}{*}{ Age category } & $10-14$ & $44(43.6 \%)$ & $88(44.9 \%)$ \\
\hline & $15-19$ & $57(56.4 \%)$ & $108(55.1 \%)$ \\
\hline \multirow[t]{3}{*}{ Residence } & Town & $56(55.4 \%)$ & $124(63.3 \%)$ \\
\hline & Rural & $45(44.6 \%)$ & $72(36.7 \%)$ \\
\hline & Orthodox & $47(46.5 \%)$ & $92(46.9 \%)$ \\
\hline \multirow[t]{3}{*}{ Religion } & Muslim & $5(5.0 \%)$ & $27(13.8 \%)$ \\
\hline & Protestant & $45(44.6 \%)$ & $72(36.7 \%)$ \\
\hline & Catholic & & \\
\hline \multirow{2}{*}{ Caregivers Marital } & Single & $17(16.8 \%)$ & $22(11.2 \%)$ \\
\hline & Married & $43(42.6 \%)$ & $111(56.6 \%)$ \\
\hline \multirow[t]{5}{*}{ status } & Divorced & $19(18.8 \%)$ & $40(20.4 \%)$ \\
\hline & Widowed & $13(12.9 \%)$ & $19(9.7 \%)$ \\
\hline & Separated & $9(8.9 \%)$ & $4(2.0 \%)$ \\
\hline & Can't read and write & $15(15 \%)$ & $23(11.7 \%)$ \\
\hline & Able to read and write & $15(15 \%)$ & $26(13.3 \%)$ \\
\hline \multirow[t]{4}{*}{$\begin{array}{l}\text { caregivers Educational } \\
\text { status }\end{array}$} & $\begin{array}{l}\text { formal education } \\
\text { (grade1-8) }\end{array}$ & $58(57 \%)$ & $113(57.7 \%)$ \\
\hline & High school and above & $13(4.4 \%)$ & $34(17.3)$ \\
\hline & Oromo & $48(47.5 \%)$ & $99(50.5 \%)$ \\
\hline & Amhara & $21(20.8 \%)$ & $41(20.9 \%)$ \\
\hline \multirow[t]{3}{*}{ Ethnicity } & Tegaru & & $6(3.1 \%)$ \\
\hline & Sidama & $22(21.8 \%)$ & $41(20.9 \%)$ \\
\hline & Others(Gedeo, Guraghe) & & $9(4.6 \%)$ \\
\hline \multirow{2}{*}{$\begin{array}{l}\text { Caregivers Employment } \\
\text { status }\end{array}$} & Employed & $75(74.3 \%)$ & $169(86.2 \%)$ \\
\hline & Unemployed & $26(25.7 \%)$ & $27(13.8 \%)$ \\
\hline \multirow{7}{*}{$\begin{array}{l}\text { Adolescents occupational } \\
\text { status }\end{array}$} & Unemployed & 5 & $12(6.1 \%)$ \\
\hline & Drivers & $10(9.9 \%)$ & $13(6.6 \%)$ \\
\hline & Students & $46(45.5 \%)$ & $94(48.0 \%)$ \\
\hline & Daily laborers & $13(12.9 \%)$ & $33(16.8 \%)$ \\
\hline & Merchants & $16(15.8 \%)$ & $24(12.2 \%)$ \\
\hline & Commercial sex workers & $9(8.9 \%)$ & $14(7.1 \%)$ \\
\hline & Others (shoeshine etc.) & & \\
\hline \multirow{3}{*}{$\begin{array}{l}\text { Adolescents Living } \\
\text { condition }\end{array}$} & Live with family & $75(74.3 \%)$ & $140(71.4 \%)$ \\
\hline & $\begin{array}{l}\text { Live with other adult or } \\
\text { children }\end{array}$ & $9(8.9 \%)$ & $17(8.7 \%)$ \\
\hline & Live alone & $17(16.8 \%)$ & $39(19.9 \%)$ \\
\hline
\end{tabular}

\section{Clinical Characteristics of Adolescents}

Majority of cases $88(87.1 \%)$ and $164(83.7 \%)$ controls were from health centers. Fifty nine $(58.4 \%)$ cases and $109(55.6 \%)$ controls were categorized as WHO clinical stage II. All controls $196(100 \%)$ have greater than $95 \%$ self/caregiver reported adherence over past one month (table 2).

Table 2: Clinical Characteristics of Adolescent on ART in Guji zone health facilities, Ethiopia, 2018. 


\begin{tabular}{llll}
\hline Variables(N=297) & Categories & Cases (\%) & Controls (\%) \\
\hline Health Facility Types & Health center & $88(87.1 \%)$ & $164(83.7 \%)$ \\
& Hospital & $13(12.9 \%)$ & $32(16.3 \%)$ \\
WHO Clinical staging & Stage I & $27(26.7 \%)$ & $61(31.1 \%)$ \\
& Stage II & $59(58.4 \%)$ & $109(55.6 \%)$ \\
& Stage III & $13(12.9 \%)$ & $20(10.2 \%)$ \\
& Stage IV & 2 & $6(3.1 \%)$ \\
ART Drug Regimen & First line & $99(98.0 \%)$ & $191(97.4 \%)$ \\
& Second line & 2 & 5 \\
Baseline CD4 Count & $\leq 350$ cells/mm & $31(56.4 \%)$ & $60(53.6 \%)$ \\
Latest CD4 count & $>350 \mathrm{cells} / \mathrm{mm}$ & $24(43.6 \%)$ & $52(46.4 \%)$ \\
& $\leq 500 \mathrm{ells} / \mathrm{mm}$ & $26(25.7 \%)$ & $26(13.3 \%)$ \\
self-reported adherence (30 days) & $<95 \%$ & $98(97 \%)$ & 0 \\
& $>500 \mathrm{cells} / \mathrm{mm}$ & $72(71.3 \%)$ & $152(77.5 \%)$ \\
& & 3 & $196(100 \%)$ \\
\hline
\end{tabular}

\section{Reason for missing ART drugs Among Adolescents}

The reason for missing ART drugs among adolescents about 66(33.7\%) controls who have missed $\leq 2$ doses in past one month recall as well as who were adherent on their 3 and 7 days recall and all cases were responded on their reason for missing ART drugs. (figure 1).

\section{Factors Associated with Nonadherence on Binary Logistic Regression}

Unmarried/widowed/separated caregivers with COR 1.8(1.1-2.9), source of income (unemployed) with COR 2.2(1.2-3.9) and area of residence (rural) COR 1.4(.85-2.3) were found to be a candidate variables associated with non-adherence status of the adolescents from socio-demographic factors (table 3 ).

Table 3: Socio-demographic factors associated with non-adherence of Adolescent on ART in Guji zone health facilities, Ethiopia, 2018. 


\begin{tabular}{|c|c|c|c|c|c|}
\hline Variables & Categories & Cases (\%) & $\begin{array}{l}\text { Controls } \\
\text { (\%) }\end{array}$ & $\begin{array}{l}\text { COR } \\
(95 \% \mathrm{CI})\end{array}$ & P.Values \\
\hline \multirow[t]{2}{*}{ Sex } & Male & $43(42.6 \%)$ & $77(39.3 \%)$ & Reference & \\
\hline & Female & $58(57.4 \%)$ & $119(60.7 \%)$ & $\begin{array}{l}0.87(.54- \\
1.4)\end{array}$ & 0.584 \\
\hline \multirow[t]{2}{*}{ Age } & $10-14$ & $44(43.6 \%)$ & $88(44.9 \%)$ & Reference & \\
\hline & $15-19$ & $57(56.4 \%)$ & $108(55.1 \%)$ & $\begin{array}{l}0.95(.58- \\
1.5)\end{array}$ & 0.827 \\
\hline \multirow{2}{*}{$\begin{array}{l}\text { Marital status } \\
\text { of caregiver }\end{array}$} & Married & $43(42.6 \%)$ & $111(56.6 \%)$ & Reference & \\
\hline & $\begin{array}{l}\text { Single, } \\
\text { widowed/separated }\end{array}$ & $58(57.4 \%)$ & $85(43.4 \%)$ & $\begin{array}{l}1.8(1.1- \\
2.9)\end{array}$ & $0.022 *$ \\
\hline \multirow{2}{*}{$\begin{array}{l}\text { Family living } \\
\text { together }\end{array}$} & Both are alive & $51(50.4 \%)$ & $101(51.5 \%)$ & Reference & \\
\hline & One or both dead & $50(49.6 \%)$ & $95(48.5 \%)$ & $\begin{array}{l}1.04(0.64- \\
1.7)\end{array}$ & 0.866 \\
\hline \multirow[t]{2}{*}{$\begin{array}{l}\text { Adolescents } \\
\text { Educational status }\end{array}$} & $\begin{array}{l}\text { Attended formal } \\
\text { education }\end{array}$ & $71(70.3 \%)$ & $147(75 \%)$ & Reference & \\
\hline & $\begin{array}{l}\text { Not attended formal } \\
\text { education }\end{array}$ & $30(29.7 \%)$ & $49(25 \%)$ & $\begin{array}{l}1.3(0.74- \\
2.2)\end{array}$ & .385 \\
\hline \multirow{2}{*}{$\begin{array}{l}\text { Sources of } \\
\text { Income }\end{array}$} & Employed & $75(74.3 \%)$ & $169(86.2 \%)$ & Reference & \\
\hline & Unemployed & $26(25.7 \%)$ & $27(13.8 \%)$ & $\begin{array}{l}2.2(1.2- \\
3.9)\end{array}$ & $0.012 *$ \\
\hline \multirow[t]{2}{*}{ Monthly Income } & $<1000$ ЕTВ & $33(32.7 \%)$ & $72(36.7 \%)$ & Reference & \\
\hline & $>=1000 \mathrm{ETB}$ & $68(67.3 \%)$ & $124(63.3 \%)$ & $\begin{array}{l}1.2(0.72- \\
2.0)\end{array}$ & 0.48 \\
\hline \multirow[t]{2}{*}{ Living arrangement } & With Family & $75(74.3 \%)$ & $140(71.4 \%)$ & $\begin{array}{l}0.87(.50- \\
1.5)\end{array}$ & 0.606 \\
\hline & Living alone & $26(25.7 \%)$ & $56(28.6 \%)$ & Reference & \\
\hline \multirow[t]{2}{*}{ Residence } & Town & $56(55.4 \%)$ & $124(63.3 \%)$ & Reference & \\
\hline & Rural & $45(44.6 \%)$ & $72(36.7 \%)$ & $\begin{array}{l}1.4(.85- \\
2.3)\end{array}$ & $0.192 *$ \\
\hline
\end{tabular}

* P-value less than 0.25

\section{Psychosocial Related Factors Associated with Non-adherence to ART}

Psychosocial related variables that were found to be candidate variables were, not disclosing HIV status COR 4.6(2.7-7.7), adolescents without family support COR 1.9(1.1-3.4), no satisfaction to family support by adolescents COR .54(0.25-1.19), adolescents/caregivers who are not using method to remind drug taking time COR 1.5(.86-2.7), have experienced family death by adolescents with COR 1.4 (0.89-2.35), (Table 4).

Table 4: Psychosocial factors associated with non-adherence among of Adolescent on ART in Guji zone health facilities, Ethiopia, 2018. 


\begin{tabular}{|c|c|c|c|c|c|}
\hline Variables & Categories & $\begin{array}{l}\text { Cases } \\
(\%)\end{array}$ & Controls (\%) & COR (95\%CI) & $\begin{array}{l}\text { P. } \\
\text { Values }\end{array}$ \\
\hline \multirow[t]{2}{*}{ Disclosure status } & Not disclosed & $57(56.4 \%)$ & $43(22 \%)$ & $4.6(2.7-7.7)$ & $0.000^{*}$ \\
\hline & Disclosed & $44(43.6 \%)$ & $153(78 \%)$ & Reference & \\
\hline Family support & $\begin{array}{l}\text { No } \\
\text { Yes }\end{array}$ & $31(30.7 \%)$ & 360 & $1.9(1$ & $0.017 *$ \\
\hline \multirow{2}{*}{$\begin{array}{l}\text { Satisfaction to } \\
\text { support family }\end{array}$} & No & $9(8.9 \%)$ & $\begin{array}{l}160(81.6 \%) \\
30(15.3 \%)\end{array}$ & Keterence & \\
\hline & Yes & $92(91.1 \%)$ & $166(84.7 \%)$ & & \\
\hline \multirow[t]{2}{*}{ Reminder method used } & Yes & $74(73.3 \%)$ & $158(\% 80.6)$ & Reference & \\
\hline & No & $27(26.7 \%)$ & $38(19.4 \%)$ & $1.5(.86-2.7)$ & $0.148 *$ \\
\hline $\begin{array}{l}\text { Experienced family } \\
\text { death }\end{array}$ & $\begin{array}{l}\text { One or more } \\
\text { No one died }\end{array}$ & $\begin{array}{l}50(49.5 \%) \\
51(50.5 \%)\end{array}$ & $\begin{array}{l}79(40.3 \%) \\
117(59.7 \%)\end{array}$ & $\begin{array}{l}1.5(0.89-2.4) \\
\text { Reference }\end{array}$ & $0.130 *$ \\
\hline Depression status & $\begin{array}{l}\text { Have } \\
\text { depression }\end{array}$ & $45(44.5 \%)$ & $38(19.4 \%)$ & $3.3(1.9-5.7)$ & $0.000 *$ \\
\hline & No depression & $56(55.5 \%)$ & $158(80.6 \%)$ & Reference & \\
\hline $\begin{array}{l}\text { Internalized } \\
\text { status }\end{array}$ & $\begin{array}{l}\text { Experienced } \\
\text { Not }\end{array}$ & $\begin{array}{l}61(60.4 \%) \\
40(39.6 \%)\end{array}$ & $\begin{array}{l}97(49.5 \%) \\
99(50.5 \%)\end{array}$ & $\begin{array}{l}\text { 1.5(0.95-2.5) } \\
\text { Reference }\end{array}$ & $0.075^{*}$ \\
\hline Discrimination status & $\begin{array}{l}\text { Experienced } \\
\text { Not experience }\end{array}$ & $\begin{array}{l}54(53.5 \%) \\
47(46.5 \%)\end{array}$ & $\begin{array}{l}83(42.3 \%) \\
113(57.7 \%)\end{array}$ & $\begin{array}{l}\text { 1.2(0.72-1.9) } \\
\text { Reference }\end{array}$ & 0.491 \\
\hline Alcohol Drinking & \multicolumn{2}{|c|}{ Yes $\quad 41(40.6 \%)$} & $\begin{array}{l}\text { month } \\
\qquad 43(21.9 \%)\end{array}$ & $\begin{array}{l}2.4(1.4- \\
4.1)\end{array}$ & $0.001 *$ \\
\hline Cigarettes Smoking & $\begin{array}{l}60(59 \\
11(10\end{array}$ & $\begin{array}{l}.4 \%) \\
.9 \%)\end{array}$ & $\begin{array}{l}153(78.1 \%) \\
10(5 \%)\end{array}$ & $\begin{array}{l}\text { Reference } \\
2.3(0.9- \\
5.5)\end{array}$ & $0.071 *$ \\
\hline \multirow[t]{2}{*}{ Khat chewing } & $\begin{array}{l}90(90 \\
24(23\end{array}$ & $\begin{array}{l}.1 \%) \\
3.7 \%)\end{array}$ & $\begin{array}{l}186(95 \%) \\
19(9.6 \%)\end{array}$ & $\begin{array}{l}\text { Reference } \\
2.9(1.5-\end{array}$ & \multirow[t]{2}{*}{$0.002 *$} \\
\hline & $77(76$ & $.3 \%)$ & $177(90.4 \%)$ & Reference & \\
\hline
\end{tabular}

* P-value less than 0.25

\section{Health Care Delivery System Related Factors Associated with Non-Adherence}

Only adolescents who are following drugs instruction provided by health care provider half of the time or less time with COR 1.9(1.1-3.6) was found to be candidate variable associated with adherence status from health care delivery system related factors(table 5).

Table 5: Health care delivery system related factors associated with non-adherence of Adolescent on ART in Guji zone health facilities, Ethiopia, 2018. 


\begin{tabular}{|c|c|c|c|c|c|}
\hline Variables & Categories & Cases (\%) & $\begin{array}{l}\text { Controls } \\
\text { (\%) }\end{array}$ & $\begin{array}{l}\text { COR } \\
(95 \% \mathrm{CI})\end{array}$ & P.Values \\
\hline \multirow{2}{*}{$\begin{array}{l}\text { Health facility } \\
\text { distance }\end{array}$} & $<=5 \mathrm{KM}$ & $63(62.4 \%)$ & $115(62.2 \%)$ & Reference & \\
\hline & $>5 \mathrm{KM}$ & $38(37.6 \%)$ & $74(37.8 \%)$ & $\begin{array}{l}0.94(0.57- \\
1.5)\end{array}$ & 0.799 \\
\hline \multirow[t]{2}{*}{ Duration to reach $\mathrm{H} / \mathrm{F}$} & $>60$ minutes & $31(30.7 \%)$ & $51(26 \%)$ & $1.3(0.74-2.1)$ & 0.394 \\
\hline & $<=60$ minutes & $70(69.3 \%)$ & $145(74 \%)$ & Reference & \\
\hline \multirow[t]{2}{*}{ Means of travel to $\mathrm{H} / \mathrm{F}$} & Vehicles/motor & $68(67.3 \%)$ & $124(63.3 \%)$ & Reference & \\
\hline & On foot & $33(32.7 \%)$ & $72(36.7 \%)$ & $0.83(.50-1.4)$ & 0.488 \\
\hline \multirow[t]{2}{*}{ counseled } & No & 1 & 2 & & \\
\hline & Yes & $100(99 \%)$ & $194(98.9 \%)$ & & \\
\hline \multirow[t]{2}{*}{ Privacy kept } & No & 3 & 5 & & \\
\hline & Yes & $98(97 \%)$ & $191(97.4 \%)$ & & \\
\hline \multirow{2}{*}{$\begin{array}{l}\text { instruction for } \\
\text { drug }\end{array}$} & No & $44(43.6 \%)$ & $81(41.3 \%)$ & $1.1(0.67-1.8)$ & 0.711 \\
\hline & Yes & $57(56.4 \%)$ & $115(68.7 \%)$ & Reference & \\
\hline \multirow[t]{2}{*}{ Following instruction } & Mostly & $76(75.2 \%)$ & $168(85.7 \%)$ & Reference & \\
\hline & $\begin{array}{l}\text { sometimes } \\
\text { HC's }\end{array}$ & $\begin{array}{l}25(24.8 \%) \\
88(87.1 \%)\end{array}$ & $\begin{array}{l}28(14.3 \%) \\
164(83.7 \%)\end{array}$ & $\begin{array}{l}\text { 1.9(1.1-3.6) } \\
\text { Reference }\end{array}$ & $0.027 *$ \\
\hline Facility types & Hospitals & $13(12.9 \%)$ & $32(16.3 \%)$ & $1.3(0.66-2.6)$ & 0.432 \\
\hline \multirow{2}{*}{$\begin{array}{l}\text { Health } \\
\text { experience }\end{array}$} & Least & $6(6 \%)$ & $18(9.2 \%)$ & & \\
\hline & Experienced & $95(94 \%)$ & $178(90.8 \%)$ & & \\
\hline
\end{tabular}

* P-value less than 0.25

\section{Disease related factors associated with non-adherence to ART}

Two variables; latest CD4 count $\geq 500 \mathrm{~mm} /$ cells with COR $0.47(0.26-0.87)$ and adolescent who use other drugs besides ART COR 2.1(1.3-3.5) were identified from disease related factors to be candidate variables associated with non-adherence status of the adolescents ( Table 6).

Table 6: disease related factors associated with non-adherence of Adolescent on ART in Guji zone health facilities, Ethiopia, 2018. 


\begin{tabular}{|c|c|c|c|c|c|}
\hline Variables & Categories & Cases (\%) & $\begin{array}{l}\text { Controls } \\
\text { (\%) }\end{array}$ & COR(95\%CI) & P.Values \\
\hline $\begin{array}{lll}\text { ART } & \text { pills taken }\end{array}$ & 1 (One) & $62(61.4 \%)$ & $118(60.2 \%)$ & Reference & \\
\hline once & $\geq 2$ (Two) & $39(38.6 \%)$ & $78(39.8 \%)$ & $\begin{array}{l}0.95(0.58- \\
1.6)\end{array}$ & 0.843 \\
\hline Daily frequency ART & $\begin{array}{l}\text { Once } \\
\text { Twice or } \\
\text { above }\end{array}$ & $\begin{array}{l}52(51.4 \%) \\
49(48.6 \%)\end{array}$ & $\begin{array}{l}88(44.9 \%) \\
108(55.1 \%)\end{array}$ & $\begin{array}{l}\text { Reference } \\
0.77(.47-1.2)\end{array}$ & 0.282 \\
\hline $\begin{array}{l}\text { Duration on } \\
\quad \text { ART } \\
\text { Baseline CD4count }\end{array}$ & $\begin{array}{l}\leq 2 \text { years } \\
>2 \text { years } \\
<350 \text { cells } / \mathrm{mm}\end{array}$ & $\begin{array}{l}26(25.7 \%) \\
75(74.3 \%) \\
31(56.4 \%)\end{array}$ & $\begin{array}{l}51(26 \%) \\
145(74 \%) \\
60(53.6 \%)\end{array}$ & $\begin{array}{l}\text { Reference } \\
1.0(0.57-1.8) \\
\text { Reference }\end{array}$ & 0.959 \\
\hline Latest CD4 & $\begin{array}{l}\geq 350 \text { cells } / \mathrm{mm} \\
\text { Missing } \\
<500 \text { cells } / \mathrm{mm}\end{array}$ & $\begin{array}{l}24(43.6 \%) \\
46(45.5 \%) \\
26(25.7 \%)\end{array}$ & $\begin{array}{l}52(46.4 \%) \\
87(45.4 \%) \\
26(13.3 \%)\end{array}$ & $\begin{array}{l}1.1(0.58-2.1) \\
\text { Reference }\end{array}$ & 0.733 \\
\hline Count & $\geq 500$ cells $/ \mathrm{mm}$ & $72(71.3 \%)$ & $152(77.5 \%)$ & $\begin{array}{l}0.47(.26- \\
0.87)\end{array}$ & $0.017^{*}$ \\
\hline $\begin{array}{l}\text { WHO clinical } \\
\text { Staging }\end{array}$ & $\begin{array}{l}\text { Missing } \\
\text { Stage I \& II } \\
\text { Stage III \& IV }\end{array}$ & $\begin{array}{c}3 \\
86(85.1 \%) \\
15(14.9 \%)\end{array}$ & $\begin{array}{l}18(9.2 \%) \\
170(86.7 \%) \\
26(13.3 \%)\end{array}$ & $\begin{array}{l}\text { Reference } \\
1.1(0.57-2.3)\end{array}$ & 0.707 \\
\hline $\begin{array}{l}\text { Other drugs } \\
\text { besides ART }\end{array}$ & $\begin{array}{l}\text { Yes } \\
\text { No }\end{array}$ & $\begin{array}{l}44(43.7 \%) \\
57(56.3 \%)\end{array}$ & $\begin{array}{l}52(26.5 \%) \\
144(73.5 \%)\end{array}$ & $\begin{array}{l}2.1(1.3-3.5) \\
\text { Reference }\end{array}$ & $0.003 *$ \\
\hline ART regimen & $\begin{array}{l}\text { First line } \\
\text { Second line }\end{array}$ & $\begin{array}{c}99(98 \%) \\
2\end{array}$ & $\begin{array}{l}191(97.4 \%) \\
5(2 \%)\end{array}$ & & \\
\hline
\end{tabular}

*significant at $\mathrm{p}$. value less than 0.25

\section{Multivariable logistic regression Analysis}

After running backward stepwise multivariable logistic regression; Residence, Marital status of caregiver, sources of income for family, disclosure status, depression status of adolescents, uses of reminding method and uses of other drugs besides ART were found to be significantly associated with non-adherence to ART among adolescents. Being rural resident is associated with nonadherence to ART among adolescents. Adolescents who resides in rural area have 1.9 times less like to adhere to ART compared to those residing in urban AOR=1.9(1.13.6). Adolescents who lives with unemployed caregivers were 4.2 times less likely to adhere to ART compared to those with employed caregivers with AOR 4.2(2.0-8.9 (Table 7).

Table 7: Multivariable Logistic Regression Analysis of factors associated with non- adherence among of Adolescent on ART in Guji zone health facilities, Ethiopia, 2018. 


\begin{tabular}{|c|c|c|c|c|c|}
\hline Factors & Categories $(\mathrm{N}=297)$ & Cases (\%) & $\begin{array}{l}\text { Controls } \\
\text { (\%) }\end{array}$ & AOR(95\%CI) & $\begin{array}{l}\text { P. } \\
\text { Values }\end{array}$ \\
\hline \multirow[t]{2}{*}{ Residence } & Rural & $45(44.6 \%)$ & $72(36.7 \%)$ & $1.9(1.1-3.6)$ & $0.026^{* *}$ \\
\hline & Town & $56(55.4 \%)$ & $124(63.3 \%)$ & Reference & \\
\hline \multirow{2}{*}{$\begin{array}{l}\text { Uses of } \\
\text { Other drugs } \\
\quad \text { besides }\end{array}$} & Yes & $44(43.7 \%)$ & $52(26.5 \%)$ & $2.6(1.5-5.0)$ & $0.002 * *$ \\
\hline & No & $57(56.3 \%)$ & $144(73.5 \%)$ & Reference & \\
\hline \multirow{4}{*}{$\begin{array}{l}\text { ART } \\
\text { Disclosure } \\
\text { status of } \\
\text { Adolescent } \\
\text { Caregiver } \\
\text { Marital } \\
\text { status }\end{array}$} & Disclosed & $44(43.6 \%)$ & $153(78 \%)$ & Reference & \\
\hline & Not disclosed & $57(56.4 \%)$ & $43(22 \%)$ & $5(2.8-9.4)$ & $0.000 * *$ \\
\hline & Unmarried/widowed/separated & $58(57.4 \%)$ & $85(43.4 \%)$ & $2.1(1.2-3.8)$ & $\begin{array}{l}0 . \\
012 * *\end{array}$ \\
\hline & Married & $43(42.6 \%)$ & $111(56.6 \%)$ & Reference & \\
\hline \multirow{2}{*}{$\begin{array}{l}\text { Family } \\
\text { income } \\
\text { Source }\end{array}$} & Employed & $75(74.3 \%)$ & $169(86.2 \%)$ & Reference & \\
\hline & Unemployed & $26(25.7 \%)$ & $27(13.8 \%)$ & $4.2(2.0-8.9)$ & $0.000 * *$ \\
\hline Adolescents & No depres & $56(55.5 \%)$ & $158(80.6 \%)$ & Reference & \\
\hline $\begin{array}{l}\text { Depression } \\
\text { status }\end{array}$ & Have depression & $45(44.5 \%)$ & $38(19.4 \%)$ & $2.7(1.5-4.9)$ & $0.002 * *$ \\
\hline \multirow{6}{*}{$\begin{array}{l}\text { Alcohol use } \\
\text { in past one } \\
\text { month } \\
\text { Reminding } \\
\text { method } \\
\text { use } \\
\text { Internalized } \\
\text { stigma }\end{array}$} & Yes & $41(40.6 \%)$ & $43(21.9 \%)$ & $1.1(0.5-2.3)$ & 0.9 \\
\hline & No & $60(59.4 \%)$ & $153(78.1 \%)$ & Reference & \\
\hline & Yes & $74(73.3 \%)$ & $158(80.6 \%)$ & Reference & \\
\hline & No & $27(26.7 \%)$ & $38(19.4 \%)$ & $2.4(1.2-4.8)$ & $0.014^{* *}$ \\
\hline & Not experienced & $40(39.6 \%)$ & $99(50.5 \%)$ & Reference & \\
\hline & Experienced & $61(60.4 \%)$ & $97(49.5 \%)$ & $1.8(.98-3.1)$ & 0.060 \\
\hline
\end{tabular}

${ }^{* *}$ factors significantly associated with nonadherence to ART at P. Value $\leq 0.05$

\section{Discussion}

The finding of this study anticipated in determining factors associated with non-adherence among adolescents living with HIV who were on ART for greater than or equals to 3 months. We found that rural residence, marital status of the caregivers (unmarried, separated/widowed), unemployed caregivers, having reported symptom of depression, not disclosing HIV status, not using memory aid to remind medication taking time and uses of other drugs besides HAART were significantly associated with non-adherence among adolescents both on binary and multivariable logistic regression analysis.

We found significant association between non-adherence and rural residence that indicates Adolescents from rural area were 1.9 times more likely to not adhere to ART compared to urban residents. This finding has agreement with studies conducted in Uganda and Kenya $(18,19)$ as Adherence was worse among the rural compared to urban living adolescents. This finding of association between non-adherence and rural areas residence may indicate difficulties in access to health services and cost of transportation to refill their medication. Non adherence among adolescent from rural descent might emphasizes the need for more 
decentralization of ART provision to all facilities at the level of health centers that can be accessible by clients at their nearby health facilities. This underlines the importance of provision of geographically closer HIV treatment services such as through mobile clinics may offer promising returns for greater ART uptake and adherence among adolescent populations.

We also found that Adolescents whose caregivers were unmarried/widowed/separated among those who were living with care givers were 2.1 times likely to not adhere to ART compared with adolescents whose caregivers were married and living together. This finding has agreement with study conducted in Mekelle town of northern Ethiopia (20). while the study conducted in Addis Ababa reported as Adolescents with widowed caregivers were more likely to have the adherence to ART (21). The reason for nonadherence among adolescents with unmarried caregiver might be due to family support from married couples than their counterparts. These also emphasize the potential importance of family structure, caregiver support, and caregiver involvement in adolescent adherence.

A source of income for family was also another independent factor associated with nonadherence among adolescents in our study. Adolescents whose families were unemployed were 4.2 times more likely to become non-adherent compared with adolescents whose caregivers were employed. Contrary to this finding study conducted in eastern Ethiopia reported that being with employed caregivers increases the likelihood of nonadherence among adolescents (22). It was not found to be associated with ART adherence status in studies conducted in Aksum, Mekelle Northern Ethiopia and same study in Addis Ababa $(21,17,20)$ and another systematic review on adolescent in sub-Saharan Africa reported as there were no association between caregivers employment status and adherence status of the adolescents $(23,24)$. The reason for nonadherence among adolescent with unemployed care givers might be related with supports including financial and psychosocial supports provided to adolescents by caregivers. This may arise from lack awareness on the need for support by family to adolescents living with HIV and knowledge gap of unemployed care givers on HIV /AIDS and its treatment.

Disclosure of adolescents HIV status had various outcomes at the individual and interpersonal levels. At the same time, it occasionally strained adolescents' sexual relationships, although it did not always lead to rejection. Our finding highlights the importance of the disclosure status among adolescents was strongly associated with adherence status of the adolescents, adolescent who did not disclosed their HIV status were 5 times more likely to become non-adherent compared to adolescents who had disclosed their sero-status by themselves or by their caregivers. This finding of our study also has agreement with study conducted in south wollo and Aksum northern Ethiopia (17)and study conducted in Nepal southeast Asia 25) reported as not disclosing the HIV status had significantly associated with non-adherence. In support of our finding two studies conducted in Tanzania indicated that not disclosing HIV status has strongly associated with non-adherence to ART $(26,27)$. In contrary to this study conducted at Tikur Anbessa Hospital Addis Ababa Ethiopia found that clients who were not aware of their HIV sero-status were more likely to adhere to ART (28). While study conduct in Harari Regional state eastern Ethiopia found as there was no association between disclosure status and adherence to ART (29). The possible explanation for this finding might be as due to disclosure creates opportunities for adolescents to access adherence support and other forms of psychosocial support from family members, peers and health care providers. 
Depression is common among adolescents living with HIV (ALHIV) worldwide, and has been associated with non-adherence to highly active anti-retroviral therapy (HAART), leading to poor virologic suppression, drug resistance. However, few studies have explored the relationship between depression and ART adherence among adolescents in Ethiopia. We found strong association between depression status and nonadherence as adolescents who have reported as having symptom of depression were 2.7 times likely to become non-adherent compared to adolescents without reported symptoms of depression. different studies found that those patients considered as having depression have low ART medication adherence compared with patients not having the condition, studies conducted in different parts of Ethiopia in Diredawa and Harari town (30) and study conducted in Aksum town of northern Ethiopia reported as depressed clients have lower ART adherence 17). This finding also supported with other studies conducted in Rwanda and Uganda that reported as care givers reported depression status of the clients is associated with ART non-adherence $(31,32)$.

This study also revealed that uses of memory aids were also found to be associated with non-adherence. Use of communication devices as reminder tools, for example alarms, pagers, text messages and telephone calls could improve adherence to ART. Adolescents not using memory aids/ reminder methods to take medication were 2.4 times more likely to become non adherent compared to adolescents using reminding methods. This finding has an agreement with prospective study conducted in Jimma University specialized hospital southwest Ethiopia and study conducted in Nekemte referral Hospital of western Ethiopia(33,34). One of the reasons for missing ART drug in our study was simply forgetting. About $31 \%$ of the adolescent who missed their medication was due forgetting thus, uses of memory aids help adolescents and care givers to remind the medication taking schedule take/give the drug at recommended time which might in turn foster good adherence.

Another important finding of current study is uses of other drugs besides ART; we found adolescents using other drugs besides ART were 2.6 times more likely to become non-adherent compared to adolescents who were only on ART drugs. The finding is comparable with study conducted in Kenya that reported use of additional drugs beside ART increase the likelihood of non-adherence among adolescents (18). Contrary to this study conducted in Addis Ababa revealed that as uses of additional drugs cotrimoxazole besides ART increases the adherence status (35). While study conducted in south wollo of north eastern Ethiopia reported as there was no association between taking additional drugs besides ART and Adherence status (36). The possible explanation for this particular finding might be drug interaction between ART and other drugs, pill burden from non-ART and ART in combination and also the side effects both non ART and ART drugs on adolescents might lead to non-adherence among adolescents.

Finally unlike previous studies, socio-demographic factors such as sex, age did not show statistically significant associations with non-adherence of ART among adolescents. This might be due to difference in location and difference in socio economic characteristics of the study participants.

\section{Limitation of the Study}

We used to measure adolescents adherence to ART by caregivers/ self-report, which may be subject to recall bias and social desirability bias that overestimate/underestimate adherence. Generalizability of our results may be limited to the selected health facilities of the same setup, as our study participants were only those Adolescents on ART who received care during our study period. 


\section{Conclusion}

We found that, living with unmarried caregivers and being rural residents were associated with non-adherence to ART. Living with unemployed caregivers was found to be strongly associated with nonadherence to ART among adolescents from socio-demographic related factors. This study also demonstrates psychosocial related factors such as having symptom of depression, not disclosing HIV status and not using reminding methods were strongly associated with non-adherence among adolescents on ART. On the other hands uses of other drugs besides ART was found to be strongly associated with non- adherence to ART among adolescents from disease related factors.

\section{Declarations}

\section{Conflicts of Interest}

The authors state that they conceive no conflicts of interest.

\section{Authors' Contributions}

ZKE, and LAW designed, reviewed, and analyzed the study and wrote the manuscript. BIJ actively involved in data collection, data analysis, and preparation of the manuscript. All authors read and approved the final manuscript.

\section{Funding}

The authors received no financial support for the research, authorship, and/or publication of this article.

\section{Availability of data and materials}

The datasets used and/or analyzed during this study is not publicly available, but may be available from the corresponding author upon reasonable request.

\section{Ethical approval and consent to participate}

The study protocol was reviewed and approved by the Jimma University Research Institutional Review Board(IRB). Informed consent was sought from the eligible ART patients before participation in the study. Participants were informed of the study objectives, that participation was voluntary, and their right to withdraw from the study at any time.

\section{Consent for publication}

Not applicable

\section{Acknowledgments}

The authors are thankful to Jimma University, Guji Zonal Health Office, Public Health facilities and the study participants. 


\section{References}

1. WHO. Defining adherence. In: WHO, editor. 1st ed. Geneva: WHO; 2003. p. 1-27.

2. Osterberg L, Blaschke T. Adherence to Medication. 2005;487-97.

3. WHO/CIPHER Paediatric HIV Matters. A RESEARCH FOR AN AIDS FREE GENERATION: A GLOBAL RESEARCH AGENDA FOR ADOLESCENTS LIVING WITH HIV. 2017;1-4.

4. UNICEF. STRENGTHENING THE ADOLESCENT COMPONENT OF NATIONAL HIV PROGRAMMES THROUGH COUNTRY ASSESSMENTS. In: United Nations Children's Fund, Strengthening the Adolescent Component of National HIV Programmes through Country Assessments. Geneva; 2015. p. 1-27.

5. WHO. Recommendations for a public health approach and considerations for policy-makers and managers. In: HIV and Adolescents: Guidance for HIV Testing and Counselling and Care For Adolescents Living With HIV. f. Geneva: WHO; 2013. p. 1-100.

6. UNAIDS Joint United Nations Programme on HIV/AIDS. Global AIDS Updates. 2016.

7. Elizabeth Glaser Pediatric AIDS Foundation. Adolescent and HIV: Prioritization For Elizabeth Glaser Pediatric AIDS Foundation Programs Advocacy and Research. 2015. 1-12 p.

8. A.S.Susuman. SP3-6 Adolescent sexual behaviour and HIV / AIDS in Ethiopia: epidemiological perspectives These include: Email alerting service. BMJ. 2011;65(J Epidemiol Community Health August 2011).

9. Population Service international (PSI). The HIV Epidemic Among Adolescent Girls and Young Women Factors Driving Vulnerability of Girls and Young Women. washington D.C.; 2017.

10. Southern African HIV Clinicians Society. Guidelines for adherence to antiretroviral therapy in adolescents and young adults. Johannesburg: Southern African HIV Clinicians Society; 2017. 4 p.

11. Abera A, Fenti B, Tesfaye T, Balcha F. Factors Influencing Adherence to Antiretroviral Therapy among People Living With HIV / AIDS at ART Clinic in Jimma University Teaching Journal of Pharmacological Reports. 2015;1(1):1-6.

12. Arage G, Tessema GA, Kassa $\mathrm{H}$. Adherence to antiretroviral therapy and its associated factors among children at South Wollo Zone Hospitals, Northeast Ethiopia: a cross-sectional study. 2014;

13. Berhe N, Tegabu D, Alemayehu M. Effect of nutritional factors on adherence to antiretroviral therapy among HIV-infected adults: a case control study in Northern Ethiopia. 2013;

14. Guji zonal Health Department. Guji Zonal Health Department eHMIS annual monthly report,of 2009E.C(2016/17). Negelle Borana; 2017.

15. World Health Organisation. The use of antiretroviral drugs for treating and preventing hiv infection. second. Geneva: WHO; 2016.

16. Bitew BD, Berehane Y, Getahun EA, Abyu DM. Determinants of None-adherence to antiretroviral therapy among HIV-infected adults in Arba Minch General Hospital, Gamo Gofa Zone, Southern Ethiopia: A case control study. 2014;2(5):234-40.

17. Gebrezgabher BB, Kebede Y, Kindie M, Tetemke D, Abay M. Determinants to antiretroviral treatment non adherence among adult HIV / AIDS patients in northern Ethiopia. AIDS Res Ther. 2017;1-7. 
18. Mukui IN, Ng L, Williamson J, Wamicwe JN, Vakil S, Katana A, et al. Rates and Predictors of Non-Adherence to Antiretroviral Therapy among HIV-Positive Individuals in Kenya: Results from the Second Kenya AIDS Indicator Survey , 2012. 2016;271:1-15.

19. Nabukeera-barungi N, Elyanu P, Asire B, Katureebe C, Lukabwe I, Namusoke E, et al. Adherence to antiretroviral therapy and retention in care for adolescents living with HIV from 10 districts in Uganda. BMC Infect Dis. 2015;1-10.

20. Eticha T, Berhane L. Caregiver-reported adherence to antiretroviral therapy among HIV infected children in Mekelle ,. 2014;

21. Naod Firdu,\&, Fikre Enquselassie, Degu Jeren O. HIV-infected adolescents have low adherence to antiretroviral therapy: a cross-sectional study in Addis Ababa, Ethiopia. pan African Med journa. 2017;8688:1-11.

22. 22. Zegeye S SE. Adherence to Antiretroviral Therapy among Hiv-Infected Children Attending Hiwot Fana and Dil-Chora Art Clinic at Referral Hospitals in Eastern Ethiopia. J HIV Clin Sci Res. 2015;2(Figure 1):8-14.

23. Heestermans T, Browne JL, Aitken SC, Vervoort SC, Klipstein-grobusch K. Determinants of adherence to antiretroviral therapy among HIV-positive adults in sub-Saharan Africa : a systematic review. 2016;1-14.

24. Carly Hudelson LC. Factors associated with adherence to antiretroviral therapy among adolescents living in low- and middle- income countries: a systematic review. 2014;1-20.

25. Wasti SP, Teijlingen E Van, Simkhada P, Randall J, Baxter S, Kirkpatrick P, et al. Factors influencing adherence to antiretroviral treatment in Asian developing countries: a systematic review. 2012;17(1):71-81.

26. BONIPHACE IDINDILI, BONIPHACE JULLU FM and M, TANNER. A case-control study of factors associated with non-adherent to antiretroviraltherapy among HIV infected ... 2014;(July 2012).

27. Nyogea D, Mtenga S, Henning L, Franzeck FC, Glass TR, Letang E, et al. Determinants of antiretroviral adherence among HIV positive children and teenagers in rural Tanzania : a mixed methods study. 2015;113.

28. Biressaw S, Abegaz WE, Abebe M, Taye WA, Belay M. Adherence to Antiretroviral Therapy and associated factors among HIV infected children in Ethiopia : unannounced home-based pill count versus caregivers ' report. 2013;

29. Mitiku H, Abdosh T, Teklemariam Z. Factors Affecting Adherence to Antiretroviral Treatment in Harari National Regional State, Eastern Ethiopia. 2013;2013.

30. Letta S, Demissie A, Oljira L, Dessie Y. Factors associated with adherence to Antiretroviral Therapy (ART ) among adult people living with HIV and attending their clinical care , Eastern Ethiopia. 2016;(2015):1-7

31. Crane JBJ, Oyugi JH, Ragland K, Kawuma A, Musoke P, Bangsberg DR. Longitudinal Antiretroviral Adherence in HIV + Ugandan Parents and Their Children Initiating HAART in the MTCT-Plus Family Treatment Model: Role of Depression in Declining Adherence Over Time. 2009;82-91.

32. Fawzi MCS, Ng L, Kanyanganzi F, Kirk C, Bizimana J, Cyamatare F, et al. Mental Health and Antiretroviral Adherence Among Youth Living With HIV in Rwanda. 2017;138(October 2016).

33. Amberbir A, Woldemichael K, Getachew S, Girma B, Deribe K. Predictors of adherence to antiretroviral therapy among HIV-infected persons: a prospective study in Southwest Ethiopia. 2008;9:1-9.

34. Kassahun Tegegne Bidu and Zalalem KabaBabure. Barriers of adherence to antiretroviral therapy among HIV / AIDS infected persons in Nekemte referral Hospital , East Wollega, Oromia Regional State, Western. 
2018;10(May):64-76.

35. Biadgilign S, Deribew A, Amberbir A, Deribe K. Adherence to highly active antiretroviral therapy and its correlates among HIV infected pediatric patients in Ethiopia. 2008;53:1-9

36. Arage G, Tessema GA, Kassa $\mathrm{H}$. Adherence to antiretroviral therapy and its associated factors among children at South Wollo Zone Hospitals, Northeast Ethiopia: a cross-sectional study. 2014;

\section{Figures}

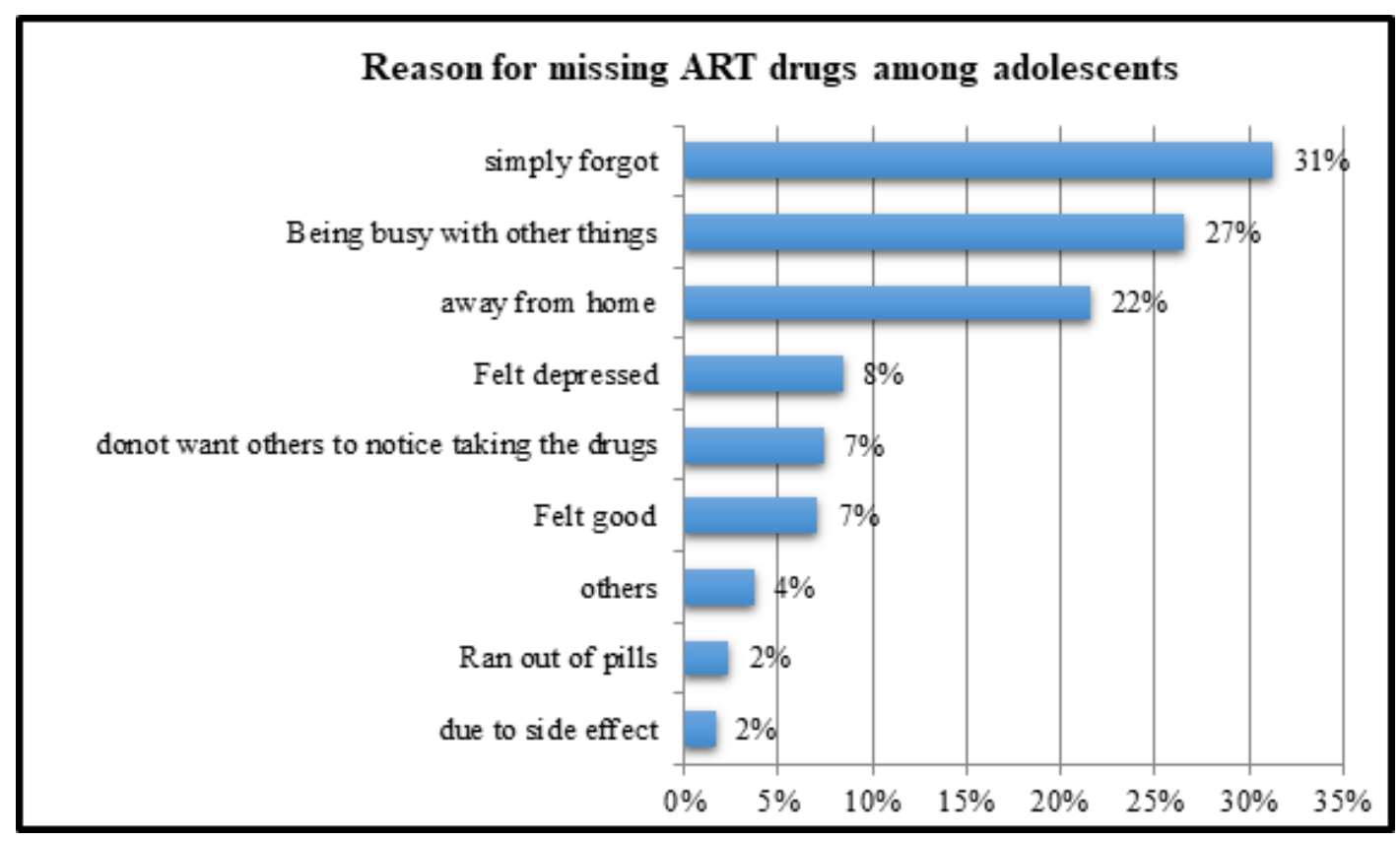

Figure 1

Reason for missing ART drug by adolescents who were on ART for greater 3 months in Guji zone, Oromia, south Ethiopia, 2018. 